\title{
Remote monitoring system based on ZigBee wireless sensor network
}

\author{
Aijuan Song, Guangyuan Si, Xiaoxiao Jiang
}

School of Control Engineering, Northeastern University at Qinhuangdao, Qinhuangdao, China

songaijuan@163

Keywords: Wireless sensor networks; ZigBee; Remote medical monitor system

\begin{abstract}
This paper deals with the monitoring system based on ZigBee wireless sensor network technique in details. The system adopts single-chipped microprocessor ZIC2410 radio frequency chip. By the upper computer software and programs of various physiological data acquisition, the physiological data of warded people, such as the pulse, blood pressure, blood oxygen saturation and electrocardiogram, are gathered by the PC software in real time, and then the collected data are transmitted to the remote medical monitoring center through the wireless sensor network. The data information of each node, including the location and health status of the warded people, can be displayed by the monitoring center software. It makes timely and effective medical guidance possible. The system is small- sized, low-power-dissipated and strongly compatible and can be connected to network with different hardware platforms. The author mainly introduces hardware circuit design of processor module, data acquisition module, and wireless communication module. The monitoring software of the host computer is developed by LabVIEW. Experiments prove that the system has good stability and security. It has very high practical value and promoting function in telemetrically monitoring field in the future.
\end{abstract}

\section{Introduction}

A Wireless sensor network (WSN) is composed of a large number of densely deployed sensor nodes, which is characterized by low-cost, high-robust, rapid deployment, self-organization. WSN has been called as a ubiquitous technology. It has wide application in military, health, smart home and medical treatment. It can be widely used in achieving the patient data, tracking treatment, mobile observation. Research on remote medical monitor system will promote the info-construction of medical treatment in China. It can speed up the development of related domains and subjects, and further enhance.

Wireless sensor networks have several applications of direct and indirect benefit to humanity, which can be further enhanced by integrating local wireless sensor networks, internet and cloud technology for storing sensors' data. The existing processes of collection and analysis of patients' information requires many efforts, but they are prone to errors with unnecessary delays in real time information accessibility. Wireless sensors provide the capability of information exchange among health monitoring equipments. The information can be stored on "cloud" and then will be accessible to medical staff. The proof-of-concept aim is to integrate commodity computing to predecessor medical devices, which should ensure simple integration and cost effectiveness into introduction of a system for health monitoring in remote areas for the best benefit of humanity [1].

M. Marzencki etc. have presented a remote personnel and asset monitoring system employing a wireless sensor network interfaced with GUI and server applications through a TCP/IP interface. The system provides users with health and activity detection, localization of user and environmental monitoring capabilities. It is more reliable than traditional hard-wired system because the system can automatically reconfigure its communication pattern when a router fails. The proposed wireless system adapts its operation to changing conditions, which is easily expandable and modular. And the system is easy and quick to be deployed at much lower cost because only minimal infrastructure is required. These results in less development and deployment time for system, and then it has lower cost and thus shorter time to market. M. Marzencki etc. also presented an example implementation of a system based on the discussed concept, which is based on the ZigBee standard and incorporates self-powered router devices with environmental and security sensors with wearable 
nodes used for measuring body position, activity level, heart rate and location of subject. So, the proposed system is innovative, low cost, and easily deployable [2].

Omeni, O. etc. presents an energy-efficient MAC Protocol designed specifically for wireless body area sensor networks focused on pervasive healthcare applications. Wireless body area networks are composed of wireless sensor nodes attached to the human body, which are used to monitor vital signs such as body temperature, heart-rate or activity. The network adopts a master-slave architecture, in which the body-worn slave node periodically sends sensor readings to a central master node. The nodes in biomedical WBASN are not deployed in an ad hoc fashion, which joining a network is centrally managed and all communications are single-hop. All the sensor nodes are in standby or sleep mode until the assigned time slot in order to reduce energy consumption. Once a node has joined a network, there is no possibility of collision within a cluster because all communication is initiated by the central node. And the communication is addressed uniquely to a slave node. A clear channel assessment algorithm based on standard listen-before-transmit is used to avoid collisions with nearby transmitters. The novel concept of a wakeup fallback time is introduced to handle time slot overlaps. Using single-hop communication and centrally controlled sleep/wakeup times leads to significant energy reductions for this application. As duty cycle is reduced, the overall power consumption approaches the standby owner. [3].

Remote medical monitoring is currently in its infancy, but its future is bright. Patients with chronic diseases will be outfitted with appropriate sensors. And data will be transported to hubs for local processing through these sensors. The data finally will be forwarded to secure medical networks for visualization and analysis by physicians, which is aided by software agents that continuously monitor the data stream. Self-sufficient patients will use this technology to stay independent longer. Then the patients in assisted-living environments can benefit from continuous monitoring and a faster, better-informed medical response to adverse events. Taking healthcare home in an inconspicuous and minimally invasive fashion equips person to be coordinated to their health, which encourages a healthier and more well-informed society [4].

\section{System Structure}

As shows, a typical remote medical monitoring system is three-tier architecture is shown in Figure 1, in which each tier distinguished by its locality and functionality within the broader system.

The first tier consists of sensors that discern signals of interest, and then information is sent to each other and the data hub.

Tier two is the data hub that provides more computational capacity. In this tier data is allowed to be stored or further processed before the data sis transmitted to outside medical network.

The third tier is the medical network operated by a hospital or telemedicine center where the staff can deal with emergency situations.

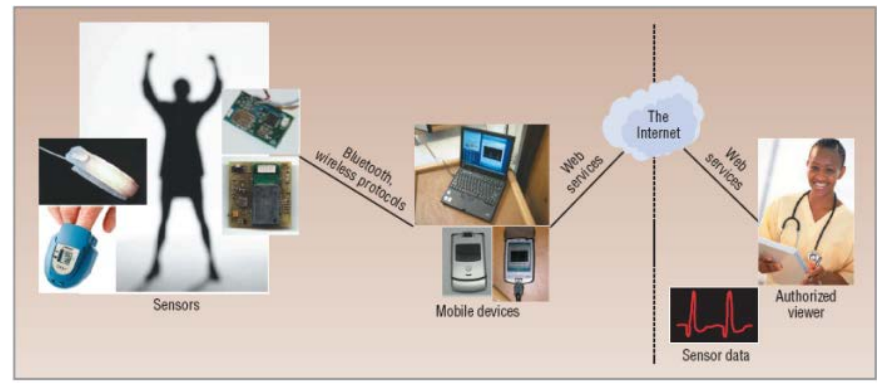

Fig.1. Remote medical monitoring system structure [4]

\section{Construction of the Wireless Monitoring System Platform}

The design of wireless remote monitoring system uses high frequency wireless transmission technology to transmit and receive information between the monitoring control instruments and 
medical sensor. As a result, connections are reduced significantly between the control equipment and sensors. Then, monitoring patient can make free activity and don't need to come back and forth between the family and the hospital. Accurate monitoring measurements can be obtained. If the wireless monitoring network covers the hospital, many clinical test projects can be completed independently without going to the inspection departments around the hospital. So, the system can bring convenience to the patients and high efficiency for the hospital. Moreover, the remote monitoring system can also monitor to other places outside of hospitals, so that some relatively and more timely medical care can be made far from the hospital patients. We can enjoy the remote guidance of doctors or doctor to some problems,

1. Monitoring System

Remote medical monitoring system is based on wireless sensor network technology. The system hardware is mainly composed of base station equipment and sensor nodes. The data from the sensor is transmitted to the medical care base station through the wireless transmission module, and then transmits the data to other network by the wireless terminal. At the same time, the data can be transmitted to a remote monitoring center through the Internet network. Statistics and analysis of the received data is achieved by professional personnel. Then the necessary related consulting services and medical guidance is provided. So, remote monitoring of medical care is realized, relative information of patients (physiological data) is sent to the monitoring center through the wireless transmission to realize the patient's immediate care and guidance. In this way, patients don’t need run between hospital and home.

This system is economic and practical. Related settings are needed based on different situation, so the system has strong maneuverability, flexibility and portability. At the same time, because the system transmission is wireless, so the limited system is easily extended. And it is more suitable for large and medium-sized medical system using for patient care and medical personnel location management etc., which can constitute a more perfect health care system. The system will find its wide use in the future.

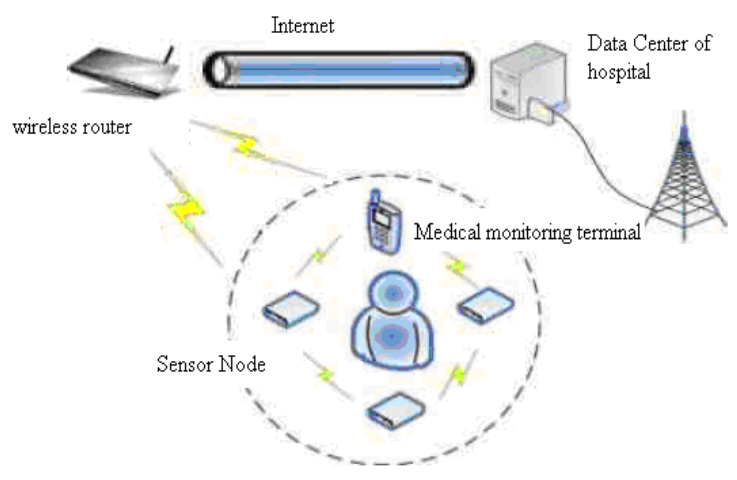

Fig.2. Monitoring system diagram

The wireless network system consists of temperature sensor DS18B20 and ECG sensor HKD-10A and respiratory sensor SL-004HKD-10A and ZigBee module. This paper mainly resolved how to collect the information heart rate (ECG) and body temperature assisted respiration signal so that more accurate analysis of patient's condition can given by the experts on the can be. The measured indexes of patients are completed by the corresponding medical sensor signal. This paper mainly collects the ECG, temperature breathing pulse signal, these sensor paste or wear in the corresponding position of the patient's body. The signal collected by the sensor by adjusting the amplification circuit, processing and analog to digital conversion, then transmits out through ZigBee transmitter module by wireless transmission to the network router.

A lot of ZigBee base station need to be set up in order not to lose data in the wireless transmission process, which are used to collect the emission signal, and then the signal is transmitted to the computer by the network coordinator.

The transmission of the signal are processed as follows, data acquisition is completed by wireless 
sensor module of each node. The data is processed by monitoring device and then transmitted ZigBee wireless module in the form of electromagnetic waves. When the wireless receiving module detects the signal at the other end of the system, the data is rapidly received, processed and sent to computer through the RS - 232 serial communication interfaces by the processor to the computer or sent to PC by a wireless module (WI-FI module) so as to realize remote control.

Remote medical monitoring system includes sensor nodes (physiological sensor and wireless ZigBee module), medical monitoring terminal (including WI-FI wireless routing module and the wireless ZigBee receiving module) and related communication equipment to connect medical monitoring network. Main problem is to solve the receiving and transmitting of wireless ZigBee module by the analysis.

\section{Management Platform Design}

Management platform mainly use software to complete the data display and monitoring function. The platform can be used to monitor physiological data on real-time and display ECG and temperature parameters so that expert can make treatment scheme. Experts and medical staff don't need back and forth between the patients and doctors' office. The platform can provide offer concrete support for $r$ the doctor's advice so as to realize the remote guidance.

1. Information management function

Information management function is to manage database information of remote medical monitoring system for managers. There are a lot of databases in the system, such as the patient database, medical staff database, medicine database and so on. The database includes the basic information of the patients and medical personnel, managers must make the corresponding update according to the change of patient and medical personnel including adding, deleting, modifying, printing and other functions.

\section{Agent function}

Receiving gateway information of the remote monitoring center is completed by agent function. When the connection is established, request agent and the responding agent will be started. It is mainly used to complete the reception, analysis and test of data from gateway at the same time control operation is completed.

\section{Diagnosis function}

The doctor can diagnose the condition of the patient through the diagnosis platform. The doctor can access the database by the diagnosis platform to search the patient information in the database, so that the doctor can rapidly make the diagnosis of patient and analyzes related physiological condition of patient, then diagnosis results are obtained according to these information. The doctor can refer the former medical record information and physiological information of patient, at same time patient can be monitored through remote control window, the current information of patient can be obtained, which is very beneficial to the doctor. The doctor can have a comprehensive understanding of the patient according to the above information. Diagnosis result can be achieved and stored in the database, which is easy for later uses. If a patient is in the wireless remote monitoring, the doctor can make diagnosis for the patient through the remote system, which brought a lot of convenience for patients, so it can be said that patients can get the guidance and the diagnosis and treatment from a doctor at home

\section{Monitoring System Software Design}

The system adopts LabVIEW as monitoring software to design the system, which uses the graphic structure and also known as the "G" language. It is standard software in data acquisition and instrument control. The advantage of the programming language doesn't need writing code, only need to connect some library function with block diagram or a flowchart, which is the unique advantage. Therefore, the idea of LabVIEW is tool for an end-user, which establish the man-machine interface and provides a lot of display or control object to meet different requirements of measurement and control field, such as knobs, header, table, such as keys, which greatly 
facilitates the software the maintenance and further development of the work.

\section{Simulation Results}

Monitoring curve of human ECG, respiration and pulse signal acquisition are shown in figure 3, figure 4 and figure 5 . According to these signal acquisition, the doctor can conveniently access real-time status of to patients.

Selected historical data column in the graph, the changes of the patient's can be judged according to current data, which is very conducive to the doctor to make rapid and effective diagnosis for the patients.

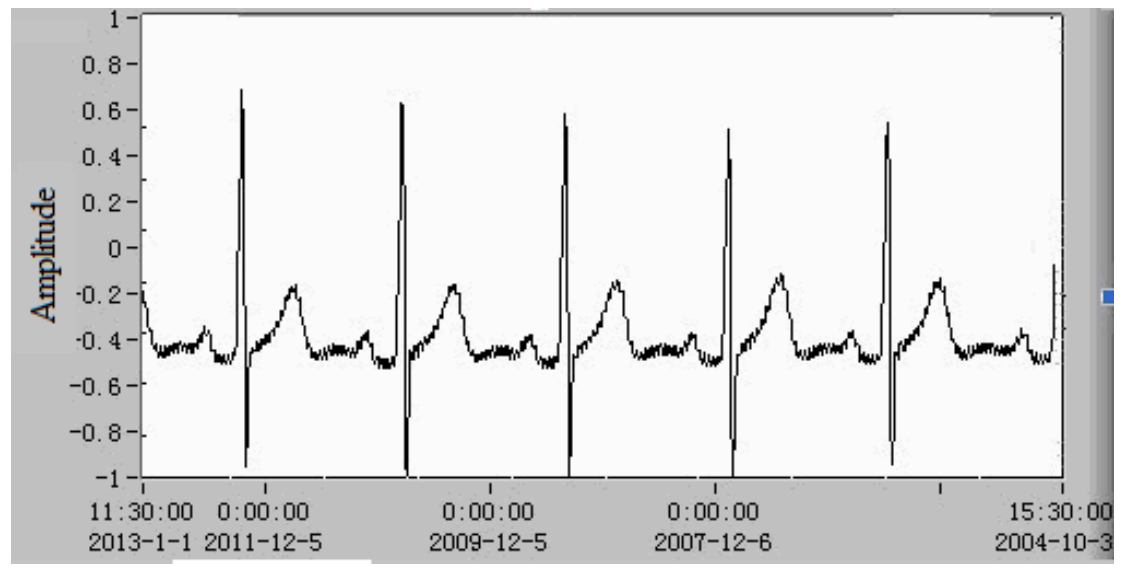

Fig.3. ECG acquisition diagram

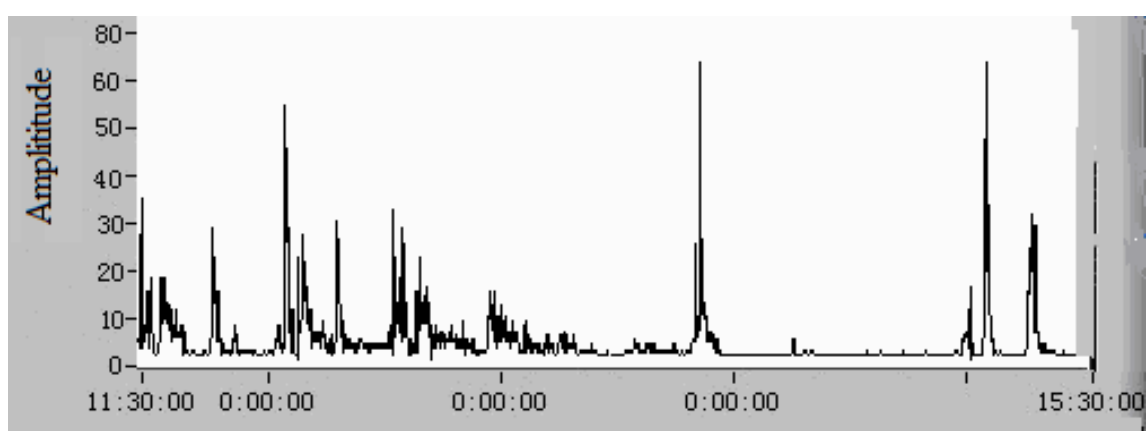

Fig.4. Breath collection diagram

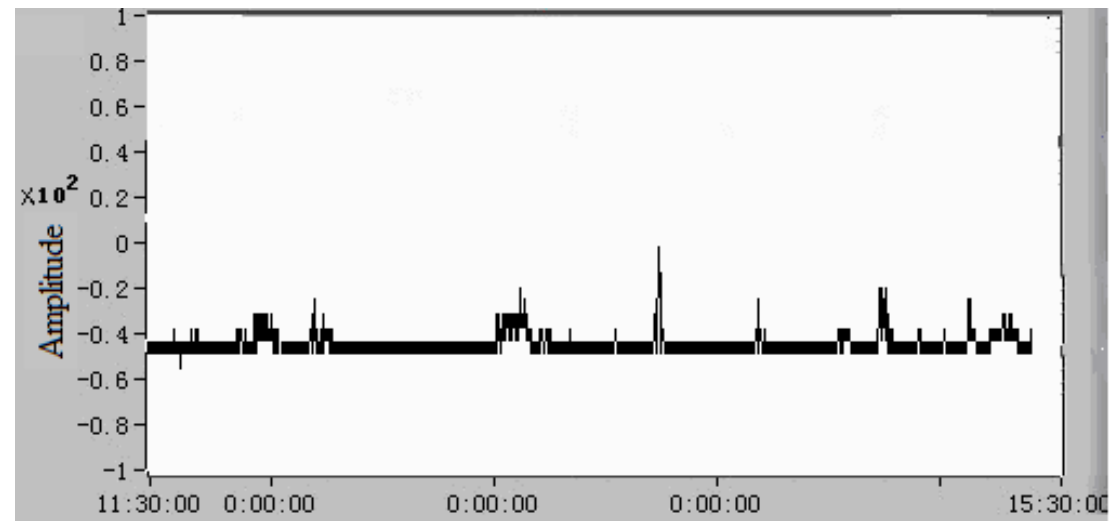

Fig.5. Pulse acquisition diagram 


\section{References}

[1] Shah, S.H., Iqbal, A., Shah, S.S.A. Remote health monitoring through an integration of wireless sensor networks, mobile phones \& Cloud Computing technologies[C], IEEE Global Humanitarian Technology Conference (GHTC), 2013: 401- 405.

[2] Marzencki, M; Lin, P. ; Cho, T.; Guo, J.; Ngai, B., Kaminska, B. Remote health, activity, and asset monitoring with wireless sensor networks[C], 13th IEEE International Conference on e-Health Networking Applications and Services (Healthcom), 2011: 98- 101.

[3] Omeni, O.,Wong, A. ,Burdett, A.J., Toumazou, C. Energy Efficient Medium Access Protocol for Wireless Medical Body Area Sensor Networks [J], IEEE Transactions on Biomedical Circuits and Systems, 2008: 2(4) 251-259.

[4] Jurik, A.D. Weaver, A.C. Remote Medical Monitoring [J], Computer, 2008:41(4) 96- 99.

[5] Song Aijuan, Study on Remote Medical System Based on Wireless Sensor Network [D], A Dissertation in Biomedical Engineering, Yanshan University. 